\title{
The Dual Behaviour of a GPCR Involved in Brain Damage an Repair: Forced Unbinding of the Receptor GPR17 Ligands from Wild Type and R255I Mutant Models Through a Computational Approach
}

\author{
Chiara Parravicini ${ }^{1}$, Maria P. Abbracchio ${ }^{1}$, Piercarlo Fantucci ${ }^{2,3}$ and Graziella Ranghino*,3 \\ ${ }^{1}$ Department of Pharmacological Sciences, Laboratory of Molecular and Cellular Pharmacology of Purinergic \\ Transmission, University of Milan, via Balzaretti 9, 20133, Milano, Italy \\ ${ }^{2}$ Department of Biotechnology and Biosciences, University of Milan-Bicocca, Piazza della Scienza 2, 20126 Milano, \\ Italy \\ ${ }^{3}$ Geol S.a.s e Delos S.r.l., via Lurani 12, 20091, Bresso, Italy
}

\begin{abstract}
GPR17 is a hybrid G-protein-coupled receptor activated by two unrelated ligand families, extracellular nucleotides and cysteinyl-leukotrienes, and involved in brain damage and repair. Its exploitment as a target for novel neuroreparative strategies depends on the elucidation of the molecular determinants driving binding of its ligands. We applied docking and molecular dynamics simulations to analyse the binding and the forced unbinding of two GPR17 ligands (the purinergic agonist UDP and the leukotriene receptor antagonist pranlukast) from both the wild-type receptor and a mutant model, where a basic residue hypothesized to be crucial for nucleotide binding had been mutated (R255I). Molecular dynamics suggested that GPR17 nucleotide binding pocket is enclosed between the helical bundle and EL2. The driving interaction involves R255 and the UDP phosphate moiety. Steered molecular dynamics experiments showed that the energy required to unbind UDP is higher for the wild-type receptor than for R255I. Three potential binding sites for pranlukast were found. In one of its preferential docking conformations, pranlukast tetrazole group is close to R255 and phenyl rings are placed into a subpocket highly conserved among GPCRs. Pulling forces developed to break polar and aromatic interactions of pranlukast were comparable. No differences between the wild-type receptor and the R255I receptor were found for the unbinding of pranlukast. These data suggest a crucial role for R255 in binding of nucleotides to GPR17. Aromatic interactions are instead likely to play a predominant role in the recognition of pranlukast, suggesting that two different binding subsites are present on GPR17.
\end{abstract}

Keywords: Steered molecular dynamics, GPR17, G-protein-coupled receptor, cysteinyl-leukotrienes, extracellular nucleotides, structural biology, homology modelling.

\section{INTRODUCTION}

GPR17 is a hybrid G-protein-coupled receptor (GPCR) that responds two both extracellular nucleotides (UDP and UDP-sugars) and cysteinyl-leukotrienes (cysteinyl-LTs, such as $\mathrm{LTC}_{4}, \mathrm{LTD}_{4}$ and $\mathrm{LTE}_{4}$ ), linking thus the P2Y and the CysLT receptor families. Furthermore, besides endogenous ligands, synthetic compounds typical of the two above receptor families are also active at GPR17. GPR17 activation can be contrasted by treatment with two well known P2Y antagonists, MRS2179 and cangrelor, and also by the already marketed CysLT receptor antagonists pranlukast and montelukast.

Recently, GPR17 has been demonstrated as a new promising target for therapeutic neuroreparative strategies in either acute and chronic cerebral dysfunctions. For example, in a model of focal rodent brain ischemia, its in vivo early knock-down with either pharmacological or specific antisense strategies, reduces the progression of cerebral ischemic damage, highlighting GPR17 as novel therapeutic target for ischemia [1].

*Address correspondence to this author at the Geol S.a.s e Delos S.r.l., via Lurani 12, 20091, Bresso, Italy; Tel: +393481627555;

Fax: +390289699020; E-mail: granghino@yahoo.it
Since at present this diseases still remains without a specific pharmacological treatment, molecules active as GPR17 inhibitors may represent a new class of promising anti-ischemic agents. On the other hand, more recent data have shown that GPR17 indeed has a dual and spatiotemporal-dependent role in the development and postinjury repair of damage in the brain and in spinal cord. GPR17 was also found to be activated on the adult precursor cells that initiate remyelination in the peri-lesioned area, suggesting a role in the transition between immature and myelinating oligodendrocytes. These data make GPR17 an attractive new target to foster repair not only in stroke but also in demyelinating diseases, such as multiple sclerosis [24].

Despite the fact that extracellular nucleotides and cysteinyl-LTs originate from totally independent metabolic pathways and they are known to be involved in distinct patho-physiological phenomena, several data suggest important functional interactions between the two families of signalling molecules and their receptors. For example, a cross-talk between the two families is suggested by the fact that both nucleotides and cysteinyl-LTs massively accumulate at sites of inflammation and both types of 
receptors are co-expressed in the same peripheral inflammatory cells. This evidence also shows a crossregulated response typical of the chemoattractant systems [5]. Along this line, in rat brain microglial cells, both nucleotides and cysteinyl-LTs, that are co-released as a consequence of the activation of $\mathrm{P} 2 \mathrm{Y}_{1}$ and CysLT receptors, contribute to neuroinflammation and neurodegeneration [6]. Nucleotides can also regulate, via heterologous desensitization, CysLT1 receptor activity [5] and, in parallel, the CysLT1 receptor antagonists pranlukast and montelukast can functionally influence $\mathrm{P} 2 \mathrm{Y}$ receptor signalling pathways in human monocyte/macrophage-like cells [7]. In addition, $\mathrm{P}_{2} \mathrm{Y}_{12}$ was found to be promiscuously activated by both nucleotides and $\mathrm{LTE}_{4}$ [8], further underlying the close relationship between the two families. Thus, the dual behaviour of GPR17, may represent a bridge between the two receptor families and further reinforces the pathophysiological role of their cooperation.

To fully understand the therapeutic potential of GPR17, specific ligands that do not interfere with the other P2Y or CysLT receptors are needed, given that the pharmacological profile of GPR17 overlaps with that of the already known P2Y and CysLT receptors. As a first step to the design of selective ligands, the knowledge of the structure and the recognition mechanism are mandatory. Along this line, we recently proposed a computational study of GPR17, highlighting a dual recognition mechanism for nucleotidic and leukotrienic ligands by GPR17: in fact, extracellular nucleotides and cysteinyl-LTs do not share the same binding site on GPR17. Our study, carried out on a bovine rhodopsin-based $(b \mathrm{Rh})$ model of GPR17, suggested that the primary nucleotide binding pocket in GPR17 is contained in an accessible crevice enclosed between transmembrane (TM) helices (mainly TM3, TM5, TM6 and TM7) and extracellular loop (EL) 2, in general agreement with the binding site proposed for small molecules to other class A rhodopsin-like GPCRs and for nucleotides to already known $\mathrm{P} 2 \mathrm{Y}$ receptors [9]. As for $\mathrm{P} 2 \mathrm{Y}$ receptors, within this pocket, the driving force for binding of nucleotides to GPR17 relies on the electrostatic interaction between the phosphate groups of incoming ligand and basic arginine residues, in particular the arginine at position 6.55 (R255) (Ballesteros-Weinstein index numbering-system, [10]). Based on our computational data, we also hypothesized that at the extracellular interface of the receptor, the $\mathrm{N}$-terminus $(\mathrm{Nt})$ region and EL2 and EL3 form accessory binding surfaces that could address ligands to the deeper main binding pocket. We finally proposed that GPR17 agonists and antagonists, although they occupy the same TM cavity, at microscopic level they probably do not share the same binding pocket [11]. On the other hand, concerning the leukotrienic component of GPR17, the characterization of the binding site is even more uncertain, due to the flexible nature of the ligands for which the identification of the docked conformation to the CysLT1 and CysLT2 receptors has not been yet successful elucidated.

To get more insight into the role of residues suggested to be crucial for the recognition mechanism by our previous computational data, the basic residue R6.55 of our GPR17 wild-type (WT) receptor model has been mutated to isoleucine, giving a mutant (R255I) receptor model of GPR17. The effects of this mutation on recognition nucleotides have been studied in silico, by simulating the "unbinding processes" of two docked ligands (the endogenous purinergic agonist the UDP and the leukotriene receptor antagonist pranlukast) from both the wild-type (WT) and the mutant (R255I) receptor model of GPR17. The results summarized in the present chapter are part of a more comprehensive study described in detail in reference 12. However, some new unpublished figures are also included. The comparison between the two simulations clearly showed that the energy required to force the unbinding of UDP from the WT receptor model is significantly higher than the work spent for the unbinding of the ligand from the R255I receptor. These data suggest that the same target residue (R255) could play a different role in either the recognition of distinct classes of ligands or in the modulation of receptor's activity when activated by ligands. The simulations summarized here can provide some attractive hypothesis on the unknown recognition mechanism and could thus be helpful for the planning of experimental mutagenesis studies and ligand affinity measurements [12].

\section{COMPARISON BETWEEN GPR17 AND NEW TEMPLATES}

Our MD simulations study [12] was performed on a $b \mathrm{Rh}$ based homology model of GPR17, for consistency with our previous study on GPR17, starting from a highly refined structure of the receptor [11]. Nevertheless, recently, thanks to protein engineering, new GPCR structures have been solved and become available for comparative modeling: the adenosine $A_{2 A}$ receptor $\left(A_{2 A} R\right)$; the $\beta_{2}$ adrenergic receptorFab $\left(\beta_{2} \mathrm{AR}-\mathrm{Fab}\right)$ and the $\beta_{2}$ adrenergic receptor-T4 $\left(\beta_{2} \mathrm{AR}\right.$ $\mathrm{T} 4)$; the mutated $\beta_{2}$ adrenergic receptor-(E122W)-T4 $\left(\beta_{2} \mathrm{AR}(\mathrm{E} 122 \mathrm{~W})-\mathrm{T} 4\right)$; the turkey $\beta_{1}$ adrenergic receptor $\left(\beta_{1} \mathrm{AR}\right)$ and the squid $\mathrm{Rh}$ [13-19]. The analysis of these newly published crystal GPCR structures further confirms that the TM7 core is conserved among the entire GPCR superfamily. Furthermore, between the sequences of the currently available GPCR structures, for GPR17 the best alignment score was obtained with $b \mathrm{Rh}$ (19.3 for $b \mathrm{Rh} ; 15.7$ for human $\beta_{2} \mathrm{AR}, 15.3$ for turkey $\beta_{1} \mathrm{AR}$ and 14.3 for human $A_{2 A} R$ ) that indeed still results as a good compromise for modeling GPR17 despite the lack on structural information on this receptor. To assure that the topology that we found for GPR17 was not an artifact due to the template, and also to assess if it was still reliable in view of the new GPCR structures, we compared our model with the structure of the human $A_{2 A} R$, the three structures of the human $\beta_{2} A R$ and the structure of the turkey $\beta_{1} A R$. Superimposition of the $C-\alpha$ atoms of the $A_{2} A R$, the $\beta_{2} A R-F a b$, the $\beta_{2} A R-T 4$, the $\beta_{2} \mathrm{AR}(\mathrm{E} 122 \mathrm{~W})-\mathrm{T} 4$ and the $\beta_{1} \mathrm{AR}$ to GPR17 and to $b \mathrm{Rh}$ is reported in Table 1. Root mean square deviation (RMSD) values obtained by rigidly superimposing the three structures to the GPR17 model vary from 1.95 to $2.87 \AA$, a range which is not significantly different from that obtained by superimposition of the same structures to $b \mathrm{Rh}$. Globally, the helical pack was highly conserved among all the structures, and also the alignment of the $\alpha$-helical domains to the GPR17 bundle yielded a good fit, as shown in Fig. (1).

In spite of the overall good fit among structures, there were some differences in the helical rearrangement concerning mainly TM1. Interestingly, all the available $\beta A R$ structures reveal the presence of an unexpected $\alpha$-helix domain on the EL2, that is indeed significantly different 
Table 1. Alignment Among GPR17 Model and GPCR Templates

\begin{tabular}{|c|c|c|c|c|c|}
\hline \multirow{2}{*}{ Structures } & \multicolumn{5}{|c|}{ RMSD (̊) after alignment of $\alpha$-helical carbon } \\
\hline & 3EML & 2R4R & 2RH1 & 3D4S & 2VT4 \\
\hline GPR17 & 2.69 & 2.87 & 2.46 & 2.74 & 2.4 \\
\hline$b \mathrm{Rh}$ & 1.96 & 2.21 & 2.36 & 2.28 & 2.86 \\
\hline
\end{tabular}

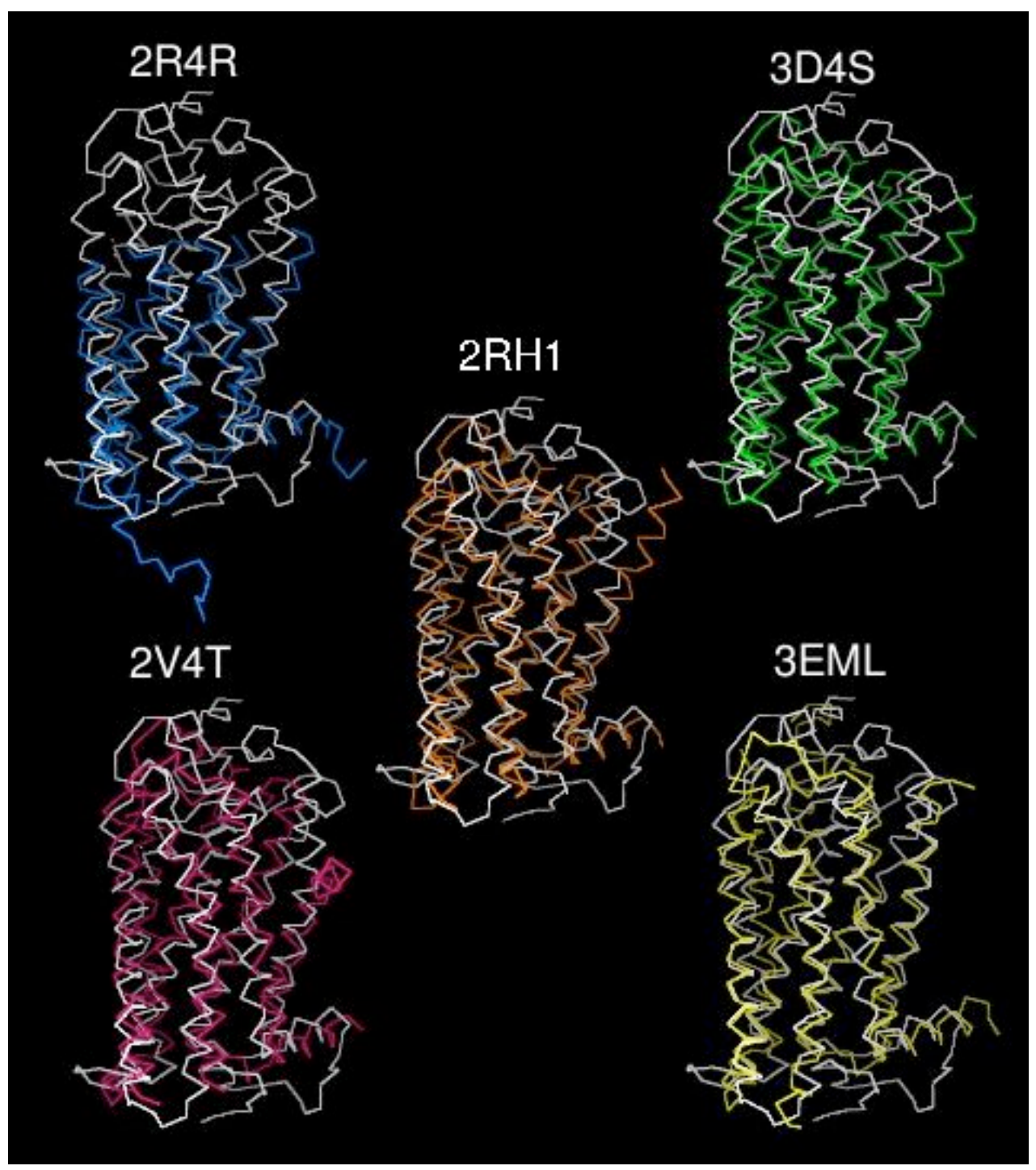

Fig. (1). Superimposition of $\alpha$-helical domains of the $\beta_{2} A R-F a b, \beta_{2} A R-T 4, \beta_{2} A R(E 122 W)-T 4, \beta_{1} A R$ and $A_{2 A}$ receptor structures to GPR17 model: Ribbon representation of the $\beta_{2} \mathrm{AR}-\mathrm{Fab}(2 \mathrm{R} 4 \mathrm{R}), \beta_{2} \mathrm{AR}-\mathrm{T} 4$ (2RH1), $\beta_{2}(\mathrm{E} 122 \mathrm{~W})-\mathrm{T} 4$ (3D4S), $\beta_{1} \mathrm{AR}(2 \mathrm{VT} 4)$ and $\mathrm{A}_{2 \mathrm{~A}}$ receptor structures after alignment of the $\alpha$-helical domains to GPR17 model (in gray) are reported in cyan, orange, green, magenta and yellow respectively.

from the $\beta$-hairpin organization that has been found for the EL2 of $b \mathrm{Rh}$, suggesting that this feature could be a requirement for the binding of reversible ligands, and that a different accessibility to the binding pocket could exist among GPCRs [20].

Moreover, the $\mathrm{A}_{2 \mathrm{~A}} \mathrm{R}$ structure reveals substantial differences in the architecture of the extracellular domains with respect to the other solved GPCR structures, as the EL2 is spatially constrained by two extra disulphide bridges that link this loop to EL1. At this time, we don't have any structural information about the macroscopic arrangement of EL2 and of the other extracellular loops in GPCRs but, being the TM bundle so well conserved during evolution, it is reasonable to assume that at least some of the keys for 


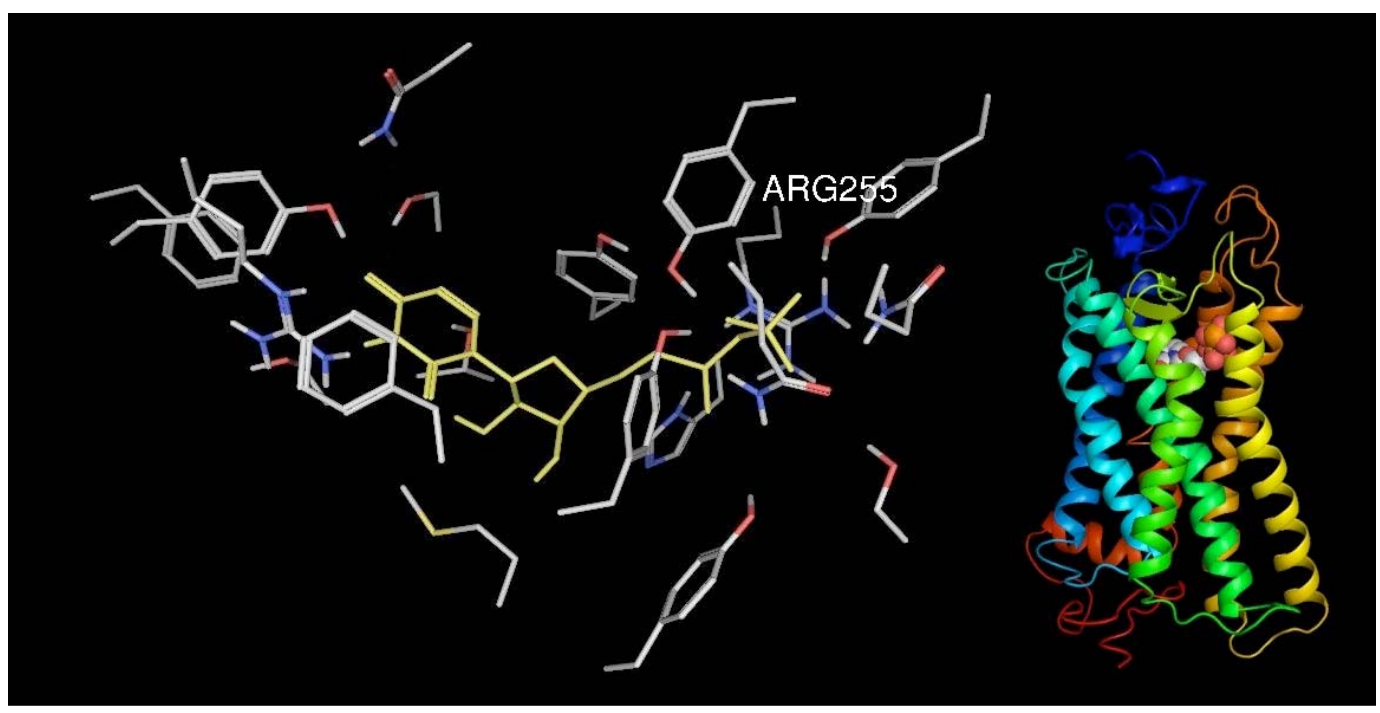

Fig. (2). Model of the best UDP conformation: GPR17-UDP complex obtained by docking and conventional MD simulation. UDP is displayed in yellow within the detailed binding pocket, and as spheres within the whole TM bundle. The interaction with R255 is highlighted.

selectivity reside in the extracellular region. This feature can also account for the exceptional plasticity of GPCRs and their capability to bind such a heterogeneous spectrum of molecules.

\section{STEERED MD SIMULATIONS}

Steered MD (SMD) experiments on GPR17-WT model, were set starting from the last minimized frame of the MD trajectory of the ligands UDP, Fig. (2), and pranlukast, Fig. (3), in their best docking configuration.

The R255 residue, that had emerged as crucial in the recognition mechanism, was mutated to isoleucine, obtaining the R255I mutant model of GPR17. SMD experiments were then performed in parallel for both the WT and the R255I mutant model complexed with the ligands; then, the mechanical resistance offered by the ligands through different unbinding pathways was measured. During the outgoing pathway from its pocket, the resistance yielded by the ligands was registered: both sterical factors and nonbonded interactions contributed to the observed peaks of force in the pulling steps. Here, for the interpretation of results, force peaks risen from SMD trajectory have been

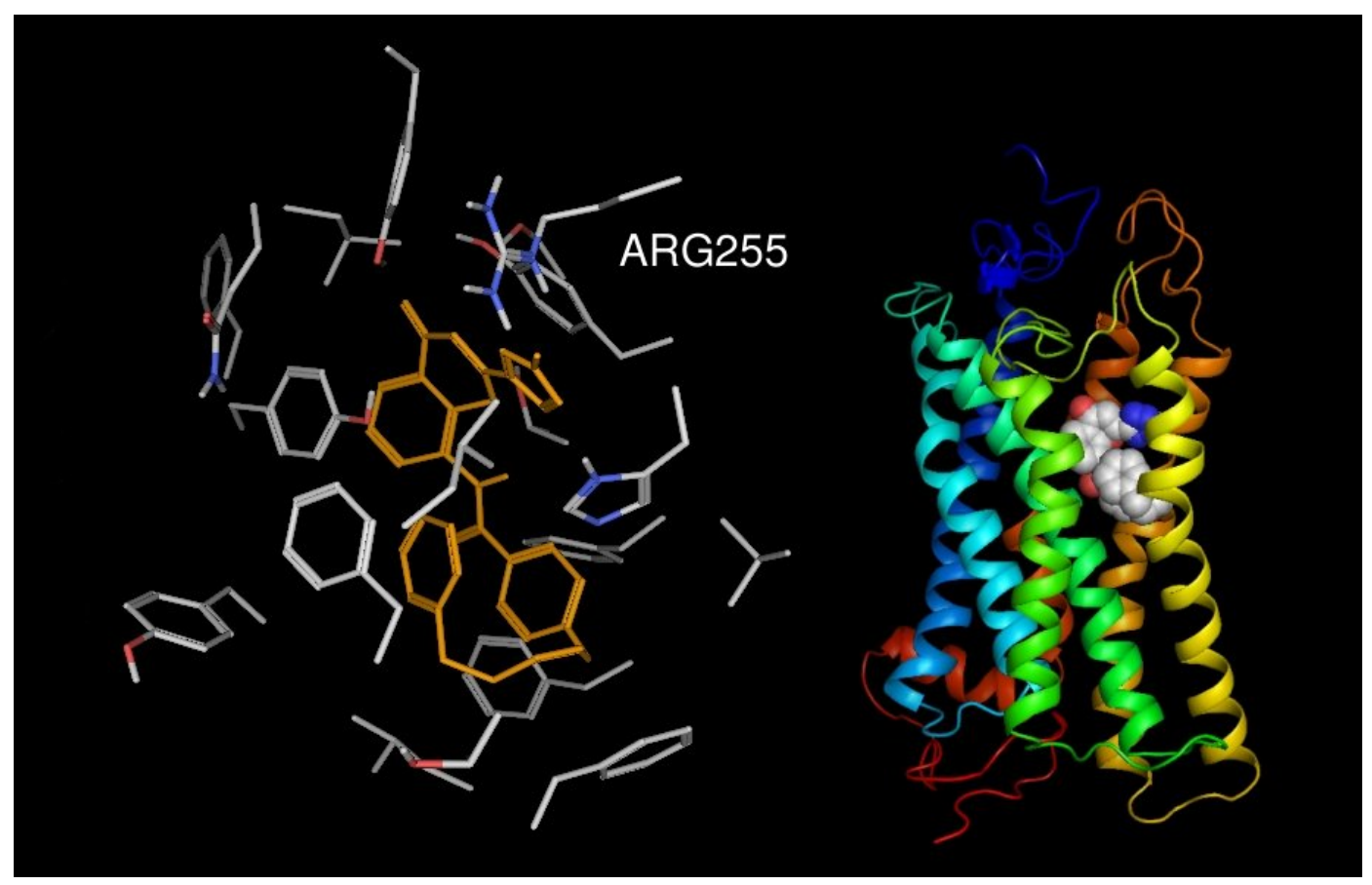

Fig. (3). Model of the best pranlukast conformation: GPR17-pranlukast complex obtained by docking and conventional MD simulation. Pranlukast is displayed in orange within the detailed binding pocket and as spheres within the whole TM bundle. The interaction with R255 is highlighted. 


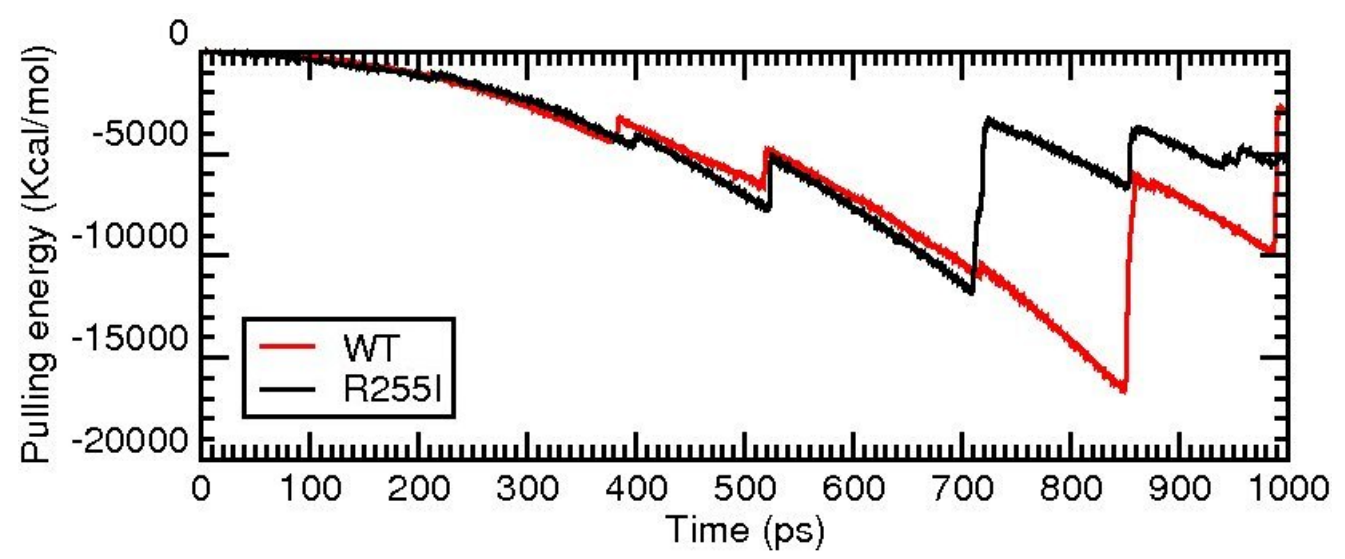

Fig. (4) Forced unbinding profile of UDP: the pulling energy developed to unbind UDP during the simulations of the forced unbinding of the ligand from the WT (in red) and the R255I (in black) receptor models are compared.

scaled to energetic values ( $\mathrm{kcal} / \mathrm{mol})$.

\section{Forced Unbinding of UDP}

In the configuration of the WT receptor, the basic residue R255 extended its sidechain across the pocket, providing the positive counterion needed to guide the interactions with the phosphate moiety typical of nucleotidic ligands, thus representing the driving forces for binding nucleotides. The architecture of the pocket of R255I-UDP complex was actually similar to that of the WT receptor in its starting configuration, but it rapidly changed during the SMD simulation, because it lacked the principal holder of the interactions network. Fig. (4) shows the SMD trajectory where the "extraction" of UDP from the WT and R255I receptor models are compared. In particular, the picture shows the work performed to pull out UDP from the WT (in red) and R255I (in black) receptor models: as expected, the energy required to unbind UDP from the WT receptor was significantly higher than that needed for the R255I receptor, suggesting that the mutation indeed affects the binding of UDP. By analyzing in detail the profile of the interactions occurring during our SMD simulations (data not shown), we found that other TM residues mainly belonging to TM3 and TM6, such as His252 (TM6), several tyrosines (Tyr112, Tyr116, Tyr120 and Tyr251) and residues belonging to extracellular regions (EL2, EL3 and Nt), also participated to the recognition of the agonist UDP. In both WT and R255I receptor, the EL2 plug constituted an energetic and sterical barrier preventing the unbinding of UDP. However, in this case, the overtaking of this barrier required less energy and took place earlier than in the WT simulation, as suggested by the comparison of the energetic profile of the pulling energy reported in Fig. (4).

\section{Forced Unbinding of Pranlukast}

As mentioned before, the SMD experiments were performed for both the WT and the R255I model on the best pranlukast docking configuration. Here, the tetrazole group of pranlukast was close to position 6.55 on TM6, and the phenyl rings were embedded into the highly conserved aromatic/hydrophobic pocket enclosed among Phe201, Phe205, Phe244 and a triplet of tyrosines belonging to TM3. In Fig. (5), the SMD unbinding trajectory of pranlukast from the WT and R255I receptors are compared.

The picture shows the pulling energy plot, computed for the WT (in red) and the R255I (in black) receptor models, respectively. No significant differences in terms of maximum value of energy were found comparing the two energetic profile for the two models; moreover, both energy profiles had values significantly lower than the ones observed for the unbinding of UDP from the WT receptor. This first observation on the energy involved in the unbinding suggests that the mutation of $\operatorname{Arg} 255$ did not

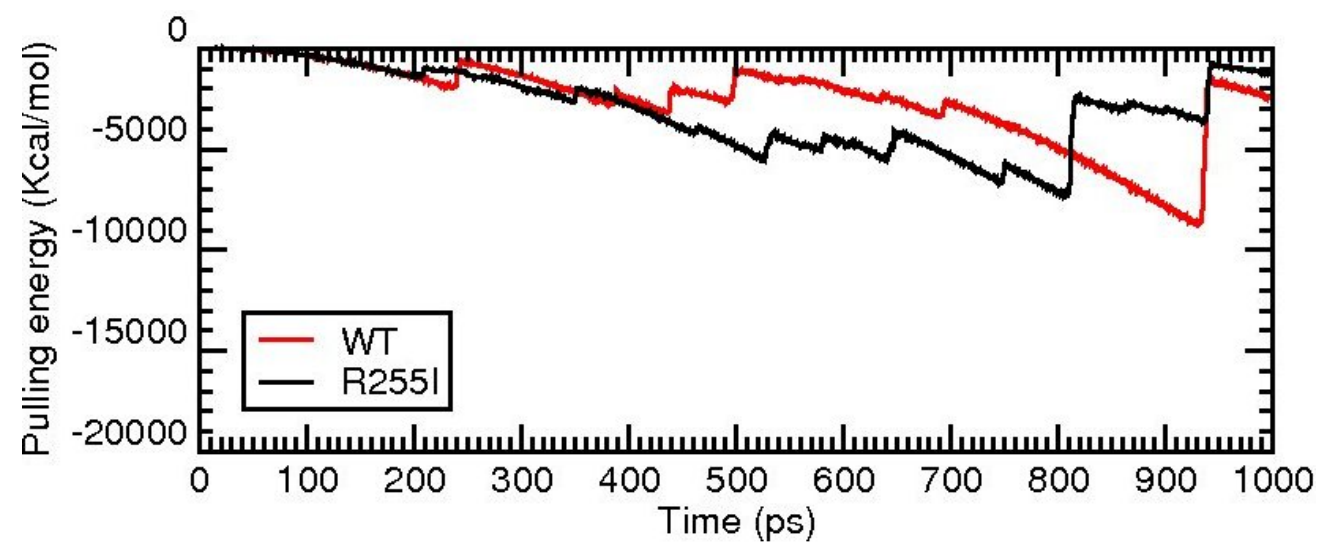

Fig. (5). Forced unbinding profile of pranlukast: the pulling energy developed to unbind pranlukast during the simulations of the forced unbinding of the ligand from the WT (in red) and the R255I (in black) receptor models are compared. 
significantly affect the binding of pranlukast to its binding pocket. The comparison of the SMD for the WT and the R255I receptor model (see panel a and b, where the WT and the R255I simulations are reported in red and black, respectively), shows only one relevant energetic peak in the case of R255I: this happened in correspondence with the transition of the ligand through the plug.

\section{EL2 BEHAVIOR}

Analysis of the SMD trajectory for both WT and R255I receptors showed that, despite the constraints imposed by the conserved disulphide bond Cys104-Cys181 linking EL2 to TM3, during the traction of the ligands out of the receptor EL2 moved toward the extracellular space showing an hinge movement that allowed the opening of the crevice on the top of the receptor. Measurement of the distance between the $\mathrm{C}$ $\alpha$ atoms of the outmost residues in both the open and closed forms of EL2 yielded to a maximum span of $6.6 \AA$, as shown in Fig. (6).

For other GPCRs, this hinge movement, that highlights the very high flexibility of EL2, has been already associated with the activation mechanism, among which the $5-\mathrm{HT}_{4 \mathrm{~A}}$ the complement factor 5a receptor $\mathrm{C} 5 \mathrm{a}$, the $\mathrm{M} 3$ and the related $\mathrm{P}_{2} \mathrm{Y}_{6}$ receptors [11].

\section{CONCLUSIONS}

Here, we summarized and implemented a computational study of a $b$ Rh-based homology model of the human GPR17 receptor, that extends our previous MD analysis of the purinergic component of this receptor and highlights some intriguing aspects of its dualistic nature. While our analysis was already in progress, the crystal structures of the first human GPCRs and of additional receptors from other species have been published [13-19]. It was therefore critical to verify that the basic structural assumptions previously made by modeling GPR17 on $b \mathrm{Rh}$ were still true at the light of the new structures. To do so, we superimposed the $\mathrm{C}-\alpha$ atoms of the $A_{2 A} R$, the $\beta_{2} A R-F a b$, the $\beta_{2} A R-T 4$, the $\beta_{2} A R(E 122 W)-$ T4 and the $\beta_{1} \mathrm{AR}$ to GPR17 and to $b \mathrm{Rh}$. The obtained RMSD values varied from 1.955 to $2.867 \AA$, a range which is not significantly different from that obtained by superimposition of the same structures to $b \mathrm{Rh}$. On this basis, we conclude that the results presented here have general value and actually give information on the putative $3 \mathrm{D}$ structure of this new receptor. This confirms that, in spite of their low sequence identity/similarity, all GPCRs share a common scaffold and supports the role of this approach as a powerful tool for the drug discovery process. However it appears clearly that the loops length and folding play a role in the ligation/recognition of the substrate.

Our data are also in line with some of the conclusions made for other GPCRs on the basis of these recently published crystal structures. For example, it has been reported that, in contrast to the $\beta$-adrenergic ligands and retinal, the $\mathrm{A}_{2 \mathrm{~A}} \mathrm{R}$ antagonist $\mathrm{ZM} 241385$ exhibits a significantly different orientation within the TM bundle. Interestingly, the bound $\mathrm{A}_{2 \mathrm{~A}} \mathrm{R}$ ligand, while interacting with helices, gets also in contact with EL2 and EL3. In a similar way, the involvement of EL2 in ligand binding to GPR17 was consistently predicted also by our SMD study, thus suggesting that this may represent a common characteristic of some specific GPCRs subgroups. This peculiarity adds diversity to the class A family of GPCRs and may play an important role in driving receptor selectivity.

Our specific challenge has been to use MD and SMD experiments as guide, to the design of in silico site-directed mutagenesis experiments that, combined with ligand affinity measurements and hopefully further structural information, will contribute to the design of new selective therapeutics for targeting GPR17. We focused our attention on Arg255, that has been proposed to play a crucial role in binding of other P2Y receptors to their nucleotide ligands [9], and substituted this Arg with Ile (R255I). Our SMD simulations showed that the energy required to unbind UDP was higher for the WT receptor model than for the mutated R255I, highlighting an important role for the basic residue also in GPR17 binding to nucleotides. No significant differences between the WT and

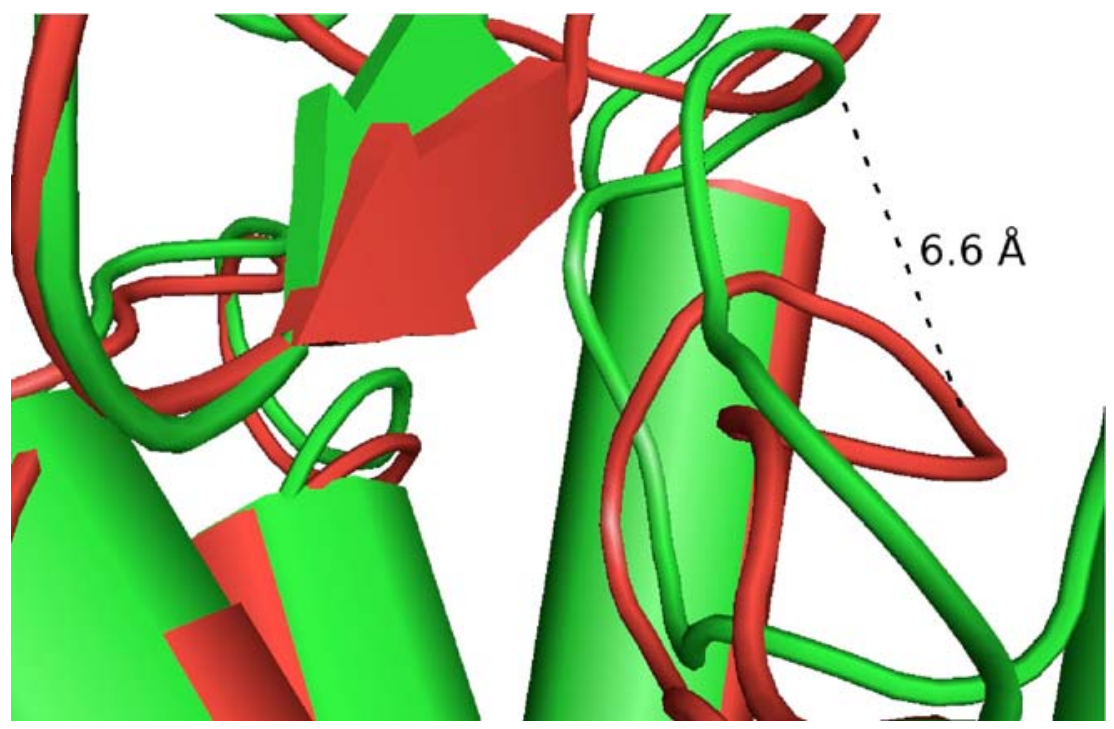

Fig. (6). EL2 dynamical behaviour: Representative frames of the open and closed form of EL2, extracted from the SMD simulations of the complex GPR17-UDP, are reported in green and red,respectively. The picture shows a detailed view of the hinge movement of the loop that exhibits an extension up to $6.6 \AA$. 
the mutated receptor were instead observed in the unbinding of the leukotrienic ligand pranlukast from GPR17; the magnitude of the forces involved was also equal to the one used to unbind UDP from the R255I mutant receptor. Furthermore, pulling forces developed to break polar and aromatic interactions of pranlukast were comparable, suggesting that aromatic interactions are likely to play a predominant role in the recognition of pranlukast. Compared with our previous data obtained simulating the forced unbinding of UDP, the magnitude of the energy used to dissociate pranlukast from both the WT and the R255I receptor models was also near to the one used to unbind UDP from the R255I mutant receptor. MD simulations thus suggest that the mutation of Arg255 influences the binding of nucleotides to GPR17, but does not affect the binding of pranlukast, indicating that two different subsites are present on GPR17 and that the intermolecular interaction networks with the ligands are different between UDP and pranlukast. This in silico data have been recently confirmed in vitro at the mutated R255I receptor [21].

The existence of two different binding sites on GPR17, regardless of the agonist and antagonist nature of the ligands, is also consistent with the intrinsic difference in the chemical structure of the two classes of unrelated purinergic and leukotrienic ligands. Moreover, this hypothesis is also supported by the peculiar organization of the TM crevice, that, in GPR17, identifies two well defined areas with different hydrophilic/phobic surface profiles. At present, the mechanism of activation and inactivation of the receptor is unknown, but some general hypothesis about the most probable target residues can be formulated, based on the present computational data. Regarding the putative nucleotide binding site, in GPR17, in agreement with the other members of the P2Y receptors family, the same binding cavity seems to be shared by purinergic agonists and antagonists, at least for small ligands. As described in our previous work [11], the antagonist cangrelor, due to its long aliphatic branches that depart from the nucleobase, can reach regions of the protein that are inaccessible to other nucleotide-derived ligands.

Concerning the leukotrienic component of GPR17, the characterization of the binding site is more uncertain, due to the flexible nature of the ligands for which the identification of the docked conformation to the CysLT1 and CysLT2 receptors has not been yet successful. Nevertheless, our data highlight the importance of the conserved aromatic/hydrophobic cluster for the recognition of pranlukast. Further investigations are needed to unveil whether this feature is shared by both agonist and antagonists. Finally, the hypothesis that two distinct binding sites, one for nucleotides and the other one leukotrienes, are present on GPR17 is in accordance with our previous experimental cross-antagonism data. It has been indeed demonstrated that, in $1321 \mathrm{~N} 1$ cells heterologously expressing hGPR17, blockade of the cysteinyl-LT binding site with the CysLT antagonists montelukast or pranlukast did not abolish the response to uracil derivatives. In a similar way, blockade of the nucleotide binding site with either cangrelor or MRS2179 still permitted the response to $\mathrm{LTD}_{4}$.

With this study, we also aimed at getting some hints on the overall mechanism of ligand recognition, i.e. not only on the role of the single amino acid residues, but also on the role played by the conformational rearrangement and mobility of protein domains, such as helices and loops. In fact, loop regions are currently deemed to be involved in the binding of large molecular weight ligands (i.e., peptides), while their role in the recognition of small moleculesresponding GPCRs remains largely unresolved. It has been proposed that EL2 does not only provide a docking surface for the recognition mechanism, but could also act as a flexible "gatekeeper" in the binding of both allosteric and orthosteric GPCR ligands [20]. In agreement with this hypothesis, our SMD simulations unmasked the flexibility of EL2, that was not evident with conventional MD simulations run in the same time scale. Globally, these data advance our knowledge on the structure of the new hybrid receptor GPR17 and will eventually contribute to the design of "dual" ligands for this new target of high therapeutic relevance.

\section{METHODS}

As highlighted above, the experiments discussed here are part of an already published original paper [12]. The reader is therefore referred to that paper for a detailed description of the Methods. Here, we provide a short summary of the key methodology mentioned in the present chapter.

\section{Steered Molecular Dynamics Simulations}

A previously published rhodopsin-based homology model of the human GPR17 receptor embedded in a hydrated dipalmitoyl-phosphatidyl-choline (DPPC) bilayer and refined by means of conventional molecular dynamics (MD) was used as starting point for both the SMD studies [11].

Docking studies and MD simulations of pranlukast and UDP were performed on the same stable 3D structure of GPR17 coming from the 10 ns MD simulations. Locally minimized structure of the ligands docked into the membrane-receptor complex subjected to conventional MD simulations, were used as starting points for SMD experiments. To induce the unbinding of ligands from the receptor, an external force was applied to center of mass (COM) of the ligand, simulating retracting cantilever directed along an imposed vector. Due to action reaction principle, the spring acts as a sensor of all interacting processes along the selected exit pathway. Pulling experiments were performed using the following parameters: pulling rate $\mathrm{v}=0.004 \mathrm{~nm} / \mathrm{ps}$ and constant force $\mathrm{k}=2000 \mathrm{~kJ} /$ mol $\mathrm{nm}^{2}$ for a total duration of $1000 \mathrm{ps}$. The vector along which ligands were pulled apart was imposed for both ligands parallel to the $z$ axis of the protein, and also parallel to the principal axis of the membrane that, for a GPCR, is likely to correspond to the only exit path possible for a bound ligand.
ABBREVIATION
3D $=$ three-dimensional
$b \mathrm{Rh} \quad=\quad$ bovine rhodopsin
$\mathrm{COM}=$ center of mass
DPPC = dipalmitoyl-phosphatidyl-choline
$\mathrm{EL}=$ extracellular loop 


$\begin{array}{lll}\text { GPCR } & \text { G-protein-coupled receptor } \\ \mathrm{LT} & =\text { cysteinyl-leukotriene } \\ \mathrm{MD} & =\text { molecular dynamics } \\ \mathrm{Nt} & =\text { N-terminus } \\ \mathrm{SMD} & =\text { steered MD } \\ \mathrm{RMSD} & =\text { root mean square deviation } \\ \mathrm{TM} & =\text { transmembrane } \\ \mathrm{WT} & =\text { wild-type }\end{array}$

\section{REFERENCES}

[1] Ciana, P.; Fumagalli, M.; Trincavelli, M.L.; Verderio, C.; Rosa, P.; Lecca, D.; Ferrario, S.; Parravicini, C.; Capra, V.; Gelosa, P.; Guerrini, U.; Belcredito, S.; Cimino, M.; Sironi, L.; Tremoli, E.; Rovati, G.E.; Martini, C.; Abbracchio, M.P.; The orphan receptor GPR17 identified as a new dual uracil nucleotides/cysteinylleukotrienes receptor. EMBO J., 2006, 25, 4615-4627.

[2] Lecca, D.; Trincavelli, M.L.; Gelosa, P.; Sironi, L.; Ciana, P.; Fumagalli, M.; Villa, G.; Verderio, C.; Grumelli, C.; Guerrini, U.; Tremoli, E.; Rosa, P.; Cuboni, S.; Martini, C.; Buffo, A.; Cimino, M.; Abbracchio, M.P. The recently identified P2Y-like receptor GPR17 is a sensor of brain damage and a new target for brain repair. PLoS One, 2008, 3, 3579.

[3] Ceruti, S.; Villa, G.; Genovese, T.; Mazzon, E.; Longhi, R.; Rosa, P.; Bramanti, P.; Cuzzocrea, S.; Abbracchio, M.P. The P2Y-like receptor GPR17 as a sensor of damage and a new potential target in spinal cord injury. Brain, 2009, 132, 2206-2218.

[4] Chen, Y.; Wu, H.; Wang, S.; Koito, H.; Li, J.; Ye, F.; Hoang, J.; Escobar, S.S.; Gow, A.; Arnett, H.A. D.; Trapp, B.D.; Karandikar, N.J.; Hsieh, J.; Lu Q.R. The oligodendrocyte-specific G proteincoupled receptor GPR17 is a cell-intrinsic timer of myelination. Nat. Neurosci., 2009, 12, 1398-1406.

[5] Capra, V.; Ravasi, S.; Accomazzo, M.R.; Citro, S.; Grimoldi, M.; Abbracchio, M.P.; Rovati, G.E. CysLT1 receptor is a target for extracellular nucleotide-induced heterologous desensitization: a possible feedback mechanism in inflammation. J. Cell Sci., 2005, 118, 5625-5636.

[6] Ballerini, P.; Di Iorio, P.; Ciccarelli, R.; Caciagli, F.; Poli, A.; Beraudi, A.; Buccella, S.; D'Alimonte, I.; D’Auro, M.; Nargi, E.; Patricelli, P.; Visini, D.; Traversa, U. P2 $\mathrm{Y}_{1}$ and cysteinyl leukotriene receptors mediate purine and cysteinyl leukotriene corelease in primary cultures of rat microglia. Int. J. Immunopathol. Pharmacol., 2005, 18, 255-268.

[7] Mamedova, L.; Capra, V.; Accomazzo, M.R.; Gao, Z.; Ferrario, S.; Fumagalli, M.; Abbracchio, M.P.; Rovati, G.E.; Jacobson, K.A. CysLT1 leukotriene receptor antagonists inhibit the effects of nucleotides acting at P2Y receptors. Biochem. Pharmacol., 2005, 71, 115-125.

[8] Nonaka, Y.; Hiramoto, T.; Fujita, N. Identification of endogenous surrogate ligands for human P2Y12 receptors by in silico and in vitro methods. Biochem. Biophys. Res. Commun., 2005, 337, 281288.
[9] Ivanov, A.A.; Costanzi, S.; Jacobson, K.A. Defining the nucleotide binding sites of P2Y receptors using rhodopsin-based homology modeling. J. Comput. Aided Mol. Des., 2006, 20, 417-426.

[10] Ballesteros, J.A.; Weinstein, H. Integrated methods for the construction of three-dimensional models of structure-funcion relations in G protein-coupled receptors. Methods Neurosci., 1995, $25,366-428$.

[11] Parravicini, C.; Ranghino, G.; Abbracchio, M.P.; Fantucci, P. GPR17: molecular modeling and dynamics studies of the 3-D structure and purinergic ligand binding features in comparison with $\mathrm{P} 2 \mathrm{Y}$ receptors. BMC Bioinformatics, 2008, 9, 263.

[12] Parravicini, C.; Abbracchio, M.P.; Fantucci, P.; Ranghino, G. Forced unbinding of GPR17 ligands from wild type and R255I mutant receptor models through a computational approach. $B M C$ Struct. Biol., 2010, 10, 8 .

[13] Jaakola, V.P.; Griffth, M.; Hanson, M.A.; Cherezov, V.; Chien, E.Y.; Lane, J.R.; Ijzerman, A.P.; Stevens, R.C. The 2.6 angstrom crystal structure of a human A2A adenosine receptor bound to an antagonist. Science, 2008, 322, 1211-1217.

[14] Rasmussen, S.G.; Choi, H.J.; Rosenbaum, D.M.; Kobilka, T.S.; Thian, F.S.; Edwards, P.C.; Burghammer, M.; Ratnala, V.R.; Sanishvili, R.; Fischetti, R.F.; Schertler, G.F.; Weis, W.I.; Kobilka, B.K. Crystal structure of the human beta2 adrenergic G-proteincoupled receptor. Nature, 2007, 450, 383-387.

[15] Cherezov, V.; Rosenbaum, D.M.; Hanson, M.A.; Rasmussen, S.G.; Thian, F.S.; Kobilka, T.S.; Choi, H.J.; Kuhn, P.; Weis, W.I.; Kobilka, B.K.; Stevens, R.C. High-resolution crystal structure of an engineered human beta2-adrenergic $\mathrm{G}$ protein-coupled receptor. Science, 2007, 318, 1258-1265.

[16] Rosenbaum, D.M.; Cherezov, V.; Hanson, M.A.; Rasmussen, S.G.; Thian, F.S.; Kobilka, T.S.; Choi, H.J.; Yao, X.J.; Weis, W.I.; Stevens, R.C.; Kobilka, B.K. GPCR engineering yields highresolution structural insights into beta2-adrenergic receptor function. Science, 2007, 318, 1266-1273.

[17] Hanson, M.A.; Cherezov, V.; Griffth, M.T.; Roth, C.B.; Jaakola, V.P.; Chien, E.Y.; Velasquez, J.; Kuhn, P.; Stevens, R.C. A specific cholesterol binding site is established by the $2.8 \mathrm{~A}$ structure of the human beta2-adrenergic receptor. Structure, 2008, $16,897-905$.

[18] Warne, T.; Serrano-Vega, M.J.; Baker, J.G.; Moukhametzianov, R.; Edwards, P.C.; Henderson, R.; Leslie, A.G.; Tate, C.G.; Schertler, G.F. Structure of a beta1-adrenergic G-protein-coupled receptor. Nature, 2008, 454, 486-491.

[19] Murakami, M.; Kouyama, T. Crystal structure of squid rhodopsin. Nature, 2008, 453, 363-367.

[20] Avlani, V.A.; Gregory, K.J.; Morton, C.J.; Parker, M.W.; Sexton, P.M.; Christopoulos, A.J. Critical role for the second extracellular loop in the binding of both orthosteric and allosteric $\mathrm{G}$ protein coupled receptor ligands. Biol. Chem., 2007, 282, 25677-25686.

[21] Calleri, E.; Ceruti, S.; Cristalli, G.; Martini, C.; Temporini, C.; Parravicini, C.; Volpini, R.; Daniele, S.; Caccialanza, G.; Lecca, D.; Lambertucci, C.; Trincavelli, M.L.; Marucci, G.; Wainer, I.W.; Ranghino, G.; Fantucci, P.; Abbracchio, M.P.; Massolini, G. Frontal affinity chromatography-mass spectrometry useful for characterization of new ligands for GPR17 receptor. J. Med. Chem., 2010, 53, 3489-3501.

(C) Parravicini et al.; Licensee Bentham Open.

This is an open access article licensed under the terms of the Creative Commons Attribution Non-Commercial License (http://creativecommons.org/licenses/by-nc/3.0/) which permits unrestricted, non-commercial use, distribution and reproduction in any medium, provided the work is properly cited. 